\title{
Herniorrafia perineal, ressecção de cisto prostático e criptorquidectomia vídeolaparoscópicas por único acesso em cão
}

\author{
Perineal vídeolaparoscopic, prostatic cystic resection, and cryptorchidectomy in a dog \\ under unique access
}

\author{
Paula Cristina Basso ${ }^{I *}$ Alceu Gaspar Raiser ${ }^{I}$ Maurício Veloso BrunII \\ Daniel Curvello de Mendonça Müller ${ }^{\mathrm{I}}$ Anelise Bonilla Trindade ${ }^{\mathrm{III}}$ Silvio Salles Cavedon ${ }^{\mathrm{IV}}$
}

\section{-NOTA-}

\section{RESUMO}

O presente relato descreve a realização de criptorquectomia, ressecção de cisto prostático e herniorrafia perineal vídeolaparoscópicas em um cão da raça Collie que apresentou recidiva dos sinais clínicos após redução cirúrgica convencional. Pela cirurgia laparoscópica, com três portais retroumbilicais, localizou-se um testículo intrabdominal, que foi removido após a aplicação de clipes de titânio no mesórquio e nos vasos testiculares. Verificou-se também a presença de um cisto prostático que foi drenado por punção transparietal com agulha $e$, em seguida, realizaram-se a ressecção da parede $e$ omentopexia na cavidade cística. Na avaliação da cavidade pélvica, verificou-se que o tamanho do defeito herniário permitia ser manejado por laparoscopia. Aplicou-se uma sutura intracorpórea no padrão colchoeiro abrangendo músculo coccígeo e elevador do ânus. O procedimento total durou aproximadamente 140 minutos, não havendo complicações. Conclui-se que a cirurgia laparoscópica pode ser empregada no tratamento de cisto prostático e na herniorrafia perineal de cães.

Palavras-chave: endocirurgia, hérnia perineal, doença prostática, caninos.

\section{ABSTRACT}

This research describes the cyst resection of the prostate, perineal hernia and laparoscopic criptorquidectomia of a Collie dog, which showed clinical signs of recurrence after conventional hernia surgery. By mean three retroumbilical portals,an intraabdominal testicle was located through laparoscopic surgery. It was removed after the application of titanium clips in mesorquio and pots testicules. A prostate cyst was noticed that was drained by puncture with a transparietal needle. An incision was made in the wall and omentopexia of the cystic cavity was performed. In the evaluation of the pelvic cavity, it was found that the hernial defect was small and could be managed by laparoscopy. An intracorporeal suturing pattern was applied which covered the coccygeal muscle and lifted the anus. The entire procedure took about 140 minutes, with no complications. It was concluded that laparoscopic surgery can be used to treat prostate cysts and the perineal hernia of dogs.

Key words: endosurgery, prostatic disease, canines.

A hérnia perineal resulta do enfraquecimento dos músculos que formam o diafragma pélvico, promovendo deslocamento caudal de órgãos abdominais ou pélvicos no períneo (MORTARI \& RAHAL, 2005). Desconhece-se a causa exata da fraqueza muscular, mas alguns fatores têm sido propostos, como a atrofia muscular neurogênica ou senil, as miopatias, o aumento de volume prostático, as alterações hormonais e a constipação crônica (HEDLUND, 2002).

Dentre as afecções prostáticas que conduzem à hérnia perineal, incluem-se os cistos

\footnotetext{
IPrograma de Pós-graduação em Medicina Veterinária (PPGMV), Centro de Ciências Rurais (CCR), Universidade Federal de Santa Maria (UFSM), Santa Maria, RS, Brasil. *Endereço para correspondência: Rua Reinaldo Manoel Guidolin, 257, Bairro Camobi, 97110-110, Santa Maria, RS, Brasil. E-mail: basso.paula@gmail.com.

IFFaculdade de Agronomia e Medicina Veterinária (FAMV), Universidade de Passo Fundo (UPF), Passo Fundo, RS, Brasil.

IIIPPGMV, CCR, Universidade Federal do Rio Grande do Sul (UFRGS), Porto Alegre, RS, Brasil.

${ }^{\text {IV } M e ́ d i c o ~ v e t e r i n a ́ r i o ~ a u t o ̂ n o m o . ~}$
} 
prostáticos. Estes são frequentemente encontrados em cães com hiperplasia prostática benigna ou outras enfermidades prostáticas concomitantes e são formados quando os canalículos dessa glândula tornam-se obstruídos, conduzindo ao acúmulo de fluído (SMITH, 2008). O tratamento pode ser realizado por meio da drenagem percutânea guiada por ultra-sonografia (BOLAND et al., 2003) ou ressecção cirúrgica do cisto (JOHNSTON et al., 2000) e do tumor testicular. Em casos mais avançados, pode ser necessária a prostatectomia parcial (RAWLINGS et al., 1997) ou total (HEDLUND, 2002).

Diversas técnicas cirúrgicas de reconstrução do diafragma pélvico são descritas na literatura. Dentre elas, destacam-se o método tradicional de sutura; a transposição do músculo obturador interno; a transposição do músculo glúteo superficial; a transposição do músculo obturador interno aliado à transposição do músculo glúteo superficial; e a transposição do músculo semitendinoso (MORTARI \& RAHAL, 2005). Além disso, também se pode recorrer ao uso de implantes sintéticos, como a membrana de látex (PAULO et al., 2005) e a malha de polipropileno (GOISSIS et al., 2001). Atualmente a herniorrafia laparoscópica tem sido utilizada com êxito em rupturas de diafragma (BECK et al., 2004) e em hérnias inguinais de cães (BRUN et al., 2006), principalmente por sua característica minimamente invasiva, que se associa à redução na dor pós-operatória e no período de recuperação (BECK et al., 2004). Entretanto, desconhece-se o uso desse acesso em hérnias perineais e cisto prostático de cães. Nesse contexto, o presente trabalho objetivou relatar um caso de criptorquidectomia, ressecção de cisto prostático e herniorrafia perineal laparoscópicas em um cão, avaliando o mesmo acesso laparoscópico na terapêutica cirúrgica das três afecções.

Para tanto, foi avaliado um cão da raça Collie, com 6,5 anos, histórico de disquesia, dor durante a defecação e que havia sido submetido à herniorrafia perineal convencional do lado esquerdo e orquiectomia do testículo em saco escrotal há trinta dias. No exame clínico, constatou-se aumento de volume redutível e indolor na região perineal esquerda. Na urinálise, verificou-se presença de bactérias e leucócitos no sedimento urinário, compatível com infecção do trato urinário inferior.

Como os sinais clínicos se mantiveram após a redução cirúrgica convencional da hérnia e considerando o possível criptorquidismo abdominal, optou-se pela cirurgia laparoscópica, com o intuito de se estabelecer o diagnóstico e tentar o tratamento. Para tanto, o paciente foi pré-medicado com maleato de acetilpromazina $\left(0,05 \mathrm{mg} \mathrm{kg}^{-1}\right.$, IM) e sulfato de morfina (0,5 $\left.\mathrm{mg} \mathrm{kg}^{-1}, \mathrm{IM}\right)$. Decorridos 15 minutos, realizou-se indução anestésica com propofol (6mg kg-1, IV). Para manutenção anestésica, foi empregado isoflurano vaporizado em $\mathrm{O}_{2}$ a 100\%, com respiração assistida.

Com o animal em decúbito dorsal, procedeuse à incisão de pele na linha média ventral retroumbilical, de aproximadamente $1 \mathrm{~cm}$, por meio da qual foi introduzida uma cânula de $10 \mathrm{~mm}$ pela técnica aberta. A cavidade foi insuflada com $\mathrm{CO}_{2}$ até a pressão de $12 \mathrm{mmHg}$. No interior da primeira cânula, introduziu-se o endoscópio rígido de zero graus e $10 \mathrm{~mm}$, sendo, em seguida, introduzidos dois trocartes adicionais nas paredes laterais direita $(10 \mathrm{~mm})$ e esquerda $(5 \mathrm{~mm})$, criando uma disposição triangular entre os portais. Iniciou-se a inspeção da cavidade abdominal onde foi verificada a presença de um cisto prostático, de aproximadamente $10 \mathrm{~cm}$ de diâmetro (Figura 1A). Localizou-se o testículo dentro da cavidade seguindo o ducto deferente, a partir da próstata. O testículo foi apreendido com uma pinça Maryland, permitindo a exposição do ducto deferente e dos vasos testiculares. Promoveu-se a aplicação de três clipes de titânio envolvendo o mesórquio e os vasos testiculares, e o testículo foi extirpado utilizando-se tesoura de Metzenbaum.

A exposição do cisto foi obtida por meio de sutura transparietal abrangendo o ápice da bexiga, sendo o ponto tracionado externamente à cavidade (Figura 1B) e fixado com pinça hemostática convencional. Após a drenagem transparietal de 120ml do conteúdo cístico translúcido com agulha e seringa, resseccionou-se a parede do cisto junto à borda da próstata, sendo utilizadas tesoura de Metzenbaum (Figura 1C) e pinça Maryland. Promoveu-se omentalização na cavidade cística, fixando-se o omento à borda residual da parede cística com um clipe de titânio (Figura 1D).

Na avaliação da cavidade pélvica, verificouse um pequeno defeito herniário, de aproximadamente dois centímetros, no lado esquerdo e procedeu-se à correção. Tracionou-se a gordura retroperitoneal herniada para o interior da cavidade abdominal e aplicou-se uma sutura intracorpórea no padrão colchoeiro em cruz com náilon monofilamentar 0 , abrangendo o músculo coccígeo e o elevador do ânus. Posteriormente, foram realizadas a drenagem do pneumoperitonio e a retirada dos trocartes. O procedimento cirúrgico foi finalizado com: obliteração da camada muscular com fio de poliglactina 910, 2-0 em padrão Sultan; do tecido subcutâneo com fio de poliglactina 910, 2-0 em sutura simples contínua; e da pele com sutura interrompida simples, sendo empregado fio mononáilon 4-0. 


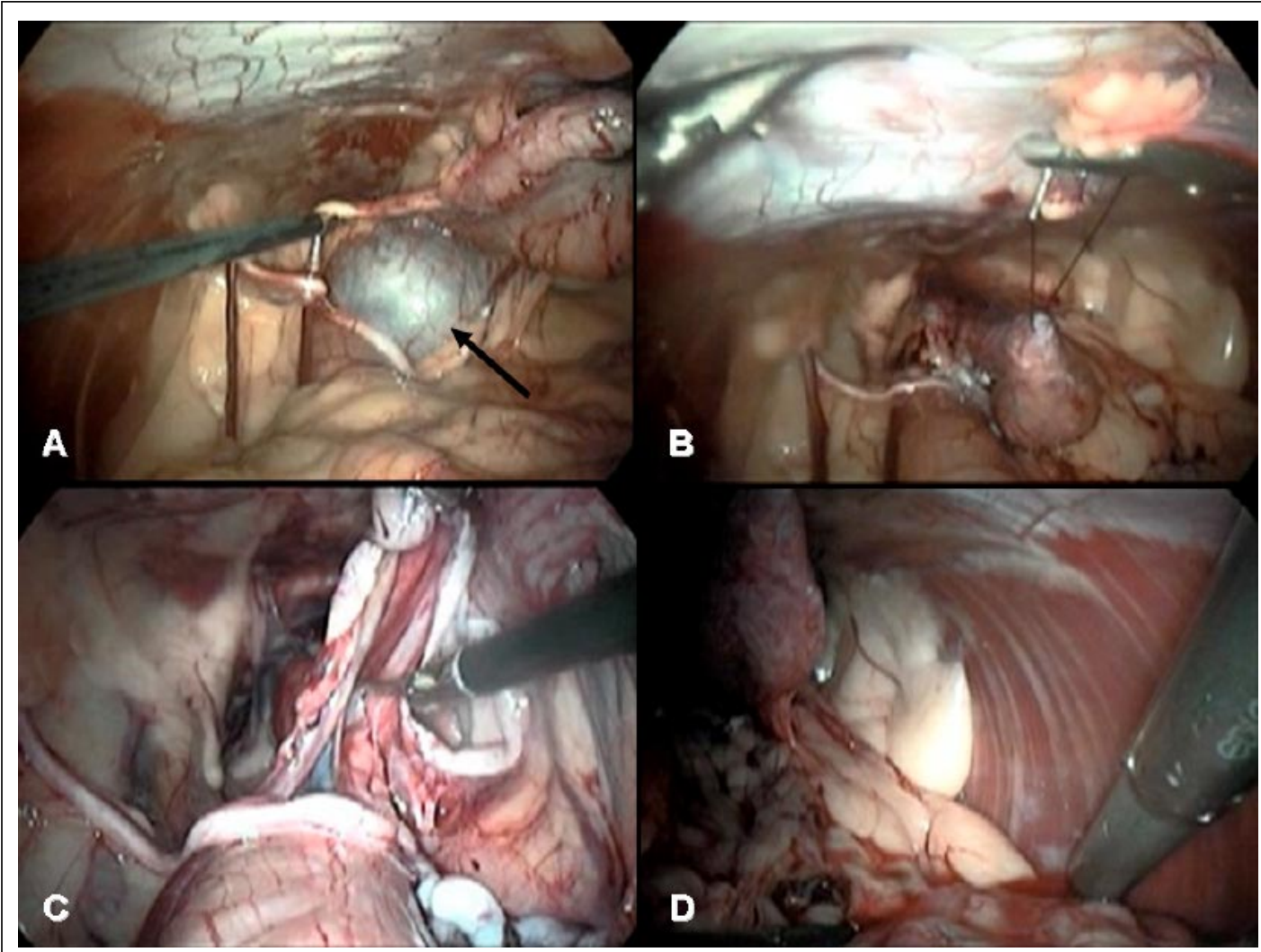

Figura 1 - Sequência da ressecção de cisto prostático por videocirurgia em um cão. (A) Visibilidade do cisto prostático. (B) Exposição do cisto obtido através de sutura transparietal passada através da parede vesical e tracionada externamente à cavidade abdominal. (C) Ressecção da parede do cisto com tesoura de Metzenbaum. (D) Imagem da região prostática omentalizada.

O procedimento total durou aproximadamente 140 minutos, sendo detectadas como únicas intercorrências transoperatórias: discreta hemorragia na região inguinal, tratada com aplicação de clipe, e formação de enfisema subcutâneo, manejado com drenagem por agulha 40×12. No pós-operatório, o paciente foi medicado com sulfato de morfina $(0,3 \mathrm{mg}$ $\mathrm{kg}^{-1}$, SC, a cada quatro horas, durante dois dias), cetoprofeno (1 $\mathrm{mg} \mathrm{kg}^{-1}$, SC, SID, durante três dias) e cefalexina (30 $\mathrm{mg} \mathrm{kg}^{-1}$, VO, BID, durante 15 dias), não apresentando sinais clínicos de alteração prostática ou herniária pelo período de 24 meses. O exame histopatológico do testículo demonstrou degeneração testicular.

Diversos fatores podem contribuir para a ocorrência de hérnias perineais; no entanto, quando associada à tenesmo, torna-se imprescindível a inclusão de doenças prostáticas e retais na lista de diagnósticos diferenciais (HEAD \& FRANCIS, 2002). Considerando essa realidade, o diagnóstico laparoscópico de cisto prostático durante a inspeção da cavidade abdominal desse animal sugere que a doença prostática primária tenha desencadeado compressão da ampola retal e do tenesmo e que o esforço persistente na defecação contribuiu para ruptura do diafragma pélvico, conforme descrito por HEAD \& FRANCIS (2002). Além disso, ainda que não tenham sido evidenciadas características neoplásicas no exame histopatológico do testículo removido, a ocorrência de retenção testicular poderia ter resultado em elevação dos níveis hormonais e desencadeado o distúrbio prostático (SPACKMAN \& ROTH, 1988).

Em comparação com a técnica tradicional para redução de hérnia perineal, citada por SOUZA \& ABÍLIO (2007), cuja incisão é aplicada sobre o tecido herniado adjacente ao ânus, considerou-se a possibilidade da laparoscopia por ser menos traumática e por apresentar menor probabilidade de infecção da ferida cirúrgica, pois evitaria a comum contaminação da área operatória com extravasamento de fezes, que acontece mesmo diante do uso de técnicas de oclusão retal (MORTARI \& RAHAL, 2005).

Com o achado laparoscópico do cisto prostático, trataram-se as três afecções por meio dos 
mesmos sítios laparoscópicos. Esse aspecto pode ser considerado importante na escolha da técnica endoscópica, em virtude de ocasionar menor lesão tecidual para a correção conjunta das doenças apresentadas. Sendo assim, a operação pode ter minimizado a perda de sangue, formação de fibrose, incidência de infecções e dor pós-operatória, as quais poderiam ter sidos observadas caso se optasse pela cirurgia convencional, conforme coloca RUDD \& HENDRICKSON (1998). Destaca-se que essa abordagem foi considerada satisfatória, sem a ocorrência de qualquer lesão iatrogênica durante a manobra. Porém, em hérnias com grandes dilatações retais, com retroflexão da bexiga ou extensa fibrose, esse procedimento pode não ser factível em razão do diminuto espaço pélvico para a realização das suturas intracorpóreas e da presença de estruturas nobres junto à área de reconstrução.

A fixação transparietal da bexiga à cavidade abdominal facilitou a visibilização prostática e a execução do procedimento sem a necessidade do posicionamento de outro portal, fato que ampliaria a lesão de acesso. Após a localização do cisto, seu conteúdo foi aspirado com auxílio de uma agulha por via percutânea, sendo a manobra visibilizada pelo endoscópio. A drenagem por punção percutânea está descrita como medida provisória para tratamento de cisto prostático, pois de fato recidivas podem acontecer (SMITH, 2008). Nesse caso, a drenagem foi instituída apenas com a finalidade de promover o esvaziamento do conteúdo cístico, para facilitar a ressecção da cápsula cística junto à próstata.

O tratamento de eleição para lesões cavitárias na próstata é a omentalização, pois, segundo FREITAG et al. (2007), resulta em baixa mortalidade pósoperatória, rápida recuperação e menor incidência de recorrência. Defende-se aqui a técnica de omentalização, pois a marsupialização, a drenagem utilizando os drenos de penrose e a prostatectomia necessitam de cuidados pós-operatórios prolongados, além de um manejo cuidadoso das feridas de drenagem (APPARÍCIO et al., 2006).

Optou-se pela omentalização da cavidade cística, com a intenção de promover diminuição do espaço morto anatômico e acreditando na ação desse tecido como um dreno fisiológico, conforme defendem APPARÍCIO et al. (2006). A prostatectomia parcial poderia ter sido realizada, como descrito por RAWLINGS et al. (1997); no entanto, a omentalização, por sua vez, apresenta baixa incidência de incontinência urinária no pós-operatório (APPARÍCIO et al., 2006) e é tecnicamente mais fácil de executar. Ao contrário da técnica de WHITE \& WILLIAMS (1995), em que o tecido prostático entre as cavidades císticas era rompido para que o omento pudesse ser introduzido de forma a circundar a uretra prostática, optou-se pelo simples preenchimento da cavidade cística com o omento e a fixação deste com grampos cirúrgicos, visando a minimizar a manipulação do órgão e causar mínima lesão no parênquima e nos componentes neurovasculares da glândula (APPARÍCIO et al., 2006). Essa característica favoreceu a melhor recuperação do animal, diminuindo o período de hospitalização.

Na medicina veterinária, apesar do número ainda reduzido, estudos descrevem a técnica laparoscópica para criptorquidectomia em cães (MILLER et al., 2004; LEW et al., 2005). Diferentemente da técnica de ligadura do cordão espermático, realizada por LEW et al. (2005), que utilizou fio absorvível, optouse pelo uso de clipes de titânio para agilizar o procedimento e minimizar o tempo cirúrgico, já que durante a exploração laparoscópica verificou-se que a orquiectomia seria apenas parte do tratamento proposto.

Com base no presente relato, conclui-se que, em casos selecionados, a terapêutica cirúrgica de criptorquidismo unilateral, cisto prostático e hérnia perineal por meio de única abordagem laparoscópica é viável e pode contribuir significativamente para a diminuição da morbidade e mortalidade pós-operatórias.

\section{REFERÊNCIAS}

APPARÍCIO, M. et al. Omentalização prostática em cães. Brazilian Journal of Veterinary Research and Animal Science, v.43, n.6, p.754-761, 2006.

BECK, C.A.C. et al. Laparoscopia nas hérnias diafragmáticas: estudo experimental em cães. Ciência Rural, v.34, n.6, p. 1849-1855, 2004. Disponível em: <http://www.scielo.br/ scielo.php? scrip t = s ci_art text \& pid = S 0103 84782004000600028>. Acesso em: 31 out. 2009. doi: 10.1590/S0103-84782004000600028.

BOLAND, L.E. et al. Ultrasound-guided percutaneous drainage as the primary treatment for prostatic abscesses and cysts in dogs. Journal of the American Animal Hospital Association, v.39, n.2, p.151-159, 2003.

BRUN, M.V. et al. Diagnóstico laparoscópico de hernias prépúbica e inguinal pós-traumática em cãe, seguido de reconstrução com traquéia homóloga conservada em glicerina. In: CONGRESSO BRASILEIRO DE CIRURGIA E ANESTESIOLOGIA VETERINÁRIA, 2006, Santos, SP. Arquivo Brasileiro de Medicina Veterinária e Zootecnia. Belo Horizonte: FEPMVZ, 2006. V.35, p.25-26.

FREITAG, T. et al. Surgical management of common canine prostatic conditions. Compendium on Continuing Education for the Veterinary Practitioner, v.29, n.11, p.656-658, 2007. 
GOISSIS, G. et al. Malhas de polipropileno recobertas com colágeno polianiônico ou com dupla camada com poli (cloreto de vinila) para reconstrução da parede abdominal. Revista Brasileira de Engenharia Biomédica, v.17, n.2, p.69-78, 2001.

HEAD, L.L.; FRANCIS, D.A. Mineralized paraprostatic cyst as a potential contributing factor in the development of perineal hernias in a dog. Journal of the American Veterinary Medical Association, v.221, n.4, p.533-555, 2002. Disponível em: <http://avmajournals.avma.org/doi/abs/10.2460/ javma.2002.221.533>. Acesso em: 31 out. 2009. doi: 10.2460/ javma.2002.221.533.

HEDLUND, C.S. Cirurgias do sistema digestivo. In: FOSSUM, T.W. Cirurgia de pequenos animais. São Paulo: Roca, 2002. Cap.16, p.389-394.

JOHNSTON, S.D. et al. Prostatic disorders in dog. Animal Reproduction Science, v.60, n.61, p.405-415, 2000.

LEW, M. et al. Laparoscopic cryptorchidectomy in dogs report of 15 cases. Polish Journal of Veterinary Sciences, v.8, n.3, p.251-254, 2005.

MILLER, N.A. et al. Use of laparoscopic-assisted cryptorchidectomy in dogs and cats. Journal of the American Veterinary Medical Association, v.224, n.6, p.875-878, 2004. Disponível em: <http://avmajournals.avma.org/doi/pdf/ 10.2460/javma.2004.224.875>. Acesso em: 31 out. 2009. doi: 10.2460/javma.2004.224.875.

MORTARI, A.C.; RAHAL, S.C. Hérnia perineal em cães. Ciência Rural, v.35, n.5, p.1220-1228, 2005. Disponível em: < http:/ /www.scielo.br/scielo.php?script=sci_arttext\&pid=S010384782005000500040 \&lang $=\mathrm{pt}>$. Acesso em: 31 out. 2009. doi: 10.1590/S0103-84782005000500040.

PAULO, N.M. et al. Biomembrana de látex natural (hevea brasilensis) com polilisina a $0,1 \%$ para herniorrafia perineal em um cão. Acta Scientiae Veterinariae, v.33, n.1, p.79-82, 2005.
RAWLINGS, C.A. et al. Use of partial prostatectomy for treatment of prostatic abscesses and cysts in dogs. Journal of the American Veterinary Medical Association, v.211, n.7, p.868-871, 1997.

RUDD, R.E.; HENDRICKSON, D.A. Minimally invasive surgery of the urinary system. In: FREEMAN, L.J. Veterinary endosurgery. St. Louis: CV Mosby, 1998. Cap.12, p.226236.

SMITH, J. Canine prostatic disease: a review of anatomy, pathology, diagnosis and treatment. Theriogenology, v.70, p.375-383, 2008. Disponível em: <http://www.sciencedirect.com/ science?_ob=ArticleURL\&_udi=B6TCM-4SN8V0F$1 \&$ _ u s e r $=687358 \&$ \& r d o c $=1 \&$ \& $\mathrm{fm} \mathrm{t}=$

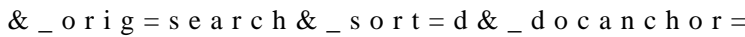
\&view=c\&_searchStrId $=1076406008 \&$ rerunOrigin $=$ google \&_acct $=$ C000037899\&_version $=1 \&$ \&_urlVersion $=0 \&$ _userid $=687358 \&$ md5=b77c7a6c50068679ec43a5db35094ae3 $>$. Acesso em: 30 out. 2009. doi: 10.1016 j.theriogenology.2008.04.039.

SOUZA, D.B.; ABÍLIO, E.J. Hérnia perineal em cães - revisão de literatura. Clínica Veterinária, n.68, p.78-86, 2007.

SPACKMAN, C.J.; ROTH, L. Prostatic cyst and concurrent sertoli cell tumor in a dog. Journal of the American Veterinary Medical Association, v.192, n.8, p.1096-1098, 1988. Disponível em: <http://www.sciencedirect.com/ science? ob=ArticleURL\&_udi=B6WWR-46SW81S 18 _user $=687358 \&$ \&doc $=1 \&$ _fmt $=$ \&_orig $=$ search\&_sort $=$ d\&_d ocanchor $=\&$ view $=$ c\&_searchStrId $=1076401319 \&$ \&rerunOrig in $=$ google\&_acct $=C 000037899 \&$ \&version $=1 \&$ \&urlVersion $=0$ \&_userid=687358\&md5=482d366789733b1bb3de02b6bea6e96b $>$. Acesso em: 31 out. 2009. doi: 10.1016/S0034-5288(02)00100-5.

WHITE, R.A.S.; WILLIAMS, J.M. Intra-capsular prostatic omentalization: a new technique for management of prostatic abscesses in dog. Veterinary Surgery, v.24, n.5, p.390-395, 1995. Disponível em: <http://www3.interscience.wiley.com/ journal/119834711/abstract?CRETRY $=1 \&$ SRETRY $=0>$. Acesso em: 30 out. 2009. doi: 10.1111/j.1532-950X.1995.tb01349.x. 